\title{
consensusDE: an R package for assessing consensus of multiple RNA-seq algorithms with RUV correction
}

\author{
Ashley J Waardenberg ${ }^{\text {Corresp., } 1}$, Matt A Field ${ }^{1,2}$ \\ ${ }^{1}$ Australian Institute for Tropical Health and Medicine, Centre for Tropical Bioinformatics and Molecular Biology, James Cook University, Smithfield, \\ Australia \\ 2 Australian National University, Canberra, Australia \\ Corresponding Author: Ashley J Waardenberg \\ Email address: a.waardenberg@gmail.com
}

Extensive evaluation of RNA-seq methods have demonstrated that no single algorithm consistently outperforms all others. Removal of unwanted variation (RUV) has also been proposed as a method for stabilizing differential expression (DE) results. Despite this, it remains a challenge to run multiple RNAseq algorithms to identify significant differences common to multiple algorithms, whilst also integrating and assessing the impact of RUV into all algorithms. consensusDE was developed to automate the process of identifying significant DE by combining the results from multiple algorithms with minimal user input and with the option to automatically integrate RUV. consensusDE only requires a table describing the sample groups, a directory containing BAM files or preprocessed count tables and an optional transcript database for annotation. It supports merging of technical replicates, paired analyses and outputs a compendium of plots to guide the user in subsequent analyses. Herein, we assess the ability of RUV to improve DE stability when combined with multiple algorithms and between algorithms, through application to real and simulated data. We find that, although RUV increased fold change stability between algorithms, demonstrated improved FDR in a setting of low replication for the intersect, the effect was algorithm specific and diminished with increased replication, reinforcing increased replication for recovery of true DE genes. We finish by offering some rules and considerations for the application of RUV in a consensus-based setting.

[p]consensusDE is freely available, implemented in R and available as a Bioconductor package, under the GPL-3 license, along with a comprehensive vignette describing functionality: http://bioconductor.org/packages/consensusDE/[p] 


\section{1 consensusDE: an R package for assessing consensus} 2 of multiple RNA-seq algorithms with RUV correction 3

4 Ashley J. Waardenberg ${ }^{1 *}$, Matt A. Field ${ }^{2}$ 5

$6 \quad{ }^{1}$ Australian Institute for Tropical Health and Medicine, Centre for Tropical Bioinformatics and

7 Molecular Biology, Centre for Molecular Therapeutics, James Cook University, Smithfield, 8 QLD 4878

$9 \quad{ }^{2}$ Australian National University, Canberra, ACT, 2612

*Corresponding Author:

13 James Cook University, PO Box 6811, Cairns, QLD, 4870, Australia

Email address: ashley.waardenberg@jcu.edu.au

Extensive evaluation of RNA-seq methods have demonstrated that no single algorithm consistently outperforms all others. Removal of unwanted variation (RUV) has also been proposed as a method for stabilizing differential expression (DE) results. Despite this, it remains a challenge to run multiple RNA-seq algorithms to identify significant differences common to multiple algorithms, whilst also integrating and assessing the impact of RUV into all algorithms. consensusDE was developed to automate the process of identifying significant DE by combining the results from multiple algorithms with minimal user input and with the option to automatically integrate RUV. consensusDE only requires a table describing the sample groups, a directory containing BAM files or preprocessed count tables and an optional transcript database for annotation. It supports merging of technical replicates, paired analyses and outputs a compendium of plots to guide the user in subsequent analyses. Herein, we assess the ability of RUV to improve DE stability when combined with multiple algorithms and between algorithms, through application to real and simulated data. We find that, although RUV increased fold for the intersect, the effect was algorithm specific and diminished with increased replication, 
33 reinforcing increased replication for recovery of true $\mathrm{DE}$ genes. We finish by offering some rules 34 and considerations for the application of RUV in a consensus-based setting.

35

36

37 38 39

40

41

42

43

44

45

46

48

49

50

consensusDE is freely available, implemented in $\mathrm{R}$ and available as a Bioconductor package, under the GPL-3 license, along with a comprehensive vignette describing functionality: http://bioconductor.org/packages/consensusDE/

\section{Introduction}

Differential gene expression (DE) analysis aims to identify transcripts or features that are expressed differently between conditions. For the detection of significant DE genes, a number of Bioconductor/R (Gentleman et al. 2004) packages have been developed that implement different statistical models for assessing DE significance. Reviews of RNA-seq DE method performance have highlighted large sensitivity and specificity differences between methods (Rapaport et al. 2013; Seyednasrollah et al. 2015). The confident selection of genes that are truly DE is especially important when trying to define reliable markers, e.g. as prognostics (Seyednasrollah et al. 2015).

Currently, there is no gold standard approach for the analysis of RNA-seq data. Whilst there remains no gold standard it is important that attention is restricted to the best performing methods, to minimise negative results that are the consequence of technical limitations. Benchmark analyses of existing algorithms for detection of DE are important for the community to assess performance of difference methods and existing analyses (Costa-Silva et al. 2017; Rapaport et al. 2013) have popularised leading algorithms, such as edgeR (McCarthy et al. 2012; Robinson et al. 2010), DESeq2 (Love et al. 2014) and limma/voom (Ritchie et al. 2015). Lin and Pang et al. recently proposed selecting a "best" method based on ranking DE stability against permutation (Lin \& Pang 2019). However, they found that no single DE method was stable in all cases, with permutation or bootstrap strategies also being limited by replicate number and computational demands. Another technique often used to assess performance of DE methods is 'False DE', where genes not expected to exhibit significant DE are examined (Rapaport et al. 2013; Seyednasrollah et al. 2015). In negative control 'False DE' experiments it was found that DE genes generally did not overlap and were specific to individual algorithms (Soneson \& Delorenzi 2013). A comparison of 11 methods found that uniquely identified DE genes are often 
65

66

67

68

69

attributed to low fold changes (Soneson \& Delorenzi 2013), but that methods largely (with some exceptions) ranked genes similarly (Soneson \& Delorenzi 2013). These findings support a "combined" or consensus-based approach or at the least a need to compare and ideally benchmark results with known truth between different methods.

Removal of unwanted sources of variation (as implemented in RUVseq), is another approach that has recently been proposed to improve DE accuracy (Risso et al. 2014). RUV aims to improve normalization, by obtaining factors that are assumed to describe unwanted variation, and subsequently including these factors in models used for DE analysis (Risso et al. 2014). Three RUV methods have been proposed, RUVr, RUVg and RUVs that utilise residuals, negative control genes or technical replicates, respectively to estimate unknown factors of random variation, given a number of unknown parameters, $\mathrm{k}$, that are subsequently incorporated into the DE model for estimation of transcript counts. RUV has been demonstrated to stabilize fold change, improving DE and separation of biological samples. Whether RUV generalizes across multiple algorithms and improves modelling in a "combined" or consensus-based setting has not been addressed to the best of our knowledge.

Implementing any consensus-based approach is challenging and requires combining individual algorithms that typically require different input parameters, use different method names, and generate different outputs - thus requiring the user to learn specific steps required for each package. Furthermore, correction methods, such as RUV (Risso et al. 2014), require users to learn additional steps for model integration. Although a number of tools have been developed for combining RNA-seq algorithms, some do not compare results from different algorithms (Delhomme et al. 2012; Varet et al. 2016), lack automation ability outside of a web-based setting (Jimenez-Jacinto et al. 2019), are not maintained in a central repository, require additional command line knowledge for installation (Costa-Silva et al. 2017), implement predecessor algorithms such as DESeq (Guo et al. 2014; Moulos \& Hatzis 2015) instead of DESeq2 (Love et al. 2014) and importantly none support RUV integration.

Integration of results from different RNA-seq algorithms would ideally allow users to easily 1) import data, 2) run RNA-seq analysis across multiple algorithms, 3) require minimal parameter input, 4) offer flexible but simple options for removal of unwanted variation (e.g. RUV integration), 5) present results together in a simple table for further analysis and finally 6) provide metrics for users to determine stability of DE calls from multiple methods. Herein, we 
96 describe consensusDE, an R/Bioconductor package, which enables the above, integrating DE

97 results from edgeR (Robinson et al. 2010), limma/voom (Ritchie et al. 2015) and DEseq2 (Love

98 et al. 2014) easily and reproducibly, with the additional option of integrating RUV. Through

99 reducing the results of multiple algorithms into a single 'consensus' table with a number of

100 descriptive statistics, users can readily assess how consistently a gene is called DE by different

101 methods and select a consensus set for further analyses. We demonstrate the utility of

102 consensusDE through application to real and simulated data and assess the impact of RUV for

103 comparability or integration with multiple RNA-seq algorithms. We find that RUV improves

104 stability of reported DE fold change and improves FDR in a setting of low replication (with

105 largest improvements for voom). However, the application of RUV did not improve true positive

106 rates (recall) or with increased number of replicates. We finish by offering some guidelines and

107 considerations for application of RUV.

108

109 Materials and Methods

110 Bioinformatics analyses were performed in R version 3.5.1 (www.r-project.org) using

111 Bioconductor (Gentleman et al. 2004) packages unless stated otherwise.

112

113 consensusDE. consensusDE version 1.3.4 (BioConductor Development version) was used for all

114 analyses. Versions of RNA-seq algorithms follow, edgeR version 3.22.5, voom (limma) version

115 3.36.5, DESeq2 version 1.20.0 and RUVSeq version 1.16.1. For DESeq2, cooksCutoff is

116 disabled for comparable reporting and ranking of all p-values. Normalisation methods are set to

117 the default methods for each algorithm in multi_de_pairs (norm_method="all_defaults"). We

118 implement RUVr, which we refer simply to RUV throughout, as described in the RUVSeq

119 BioConductor vignette (utlising edgeR and extracting residuals), with $\mathrm{k}$ set to 1 . RUVr removes

120 sources of unwanted variation, by first fitting a generalised linear model (GLM) to count data

121 and obtaining model residuals that are then incorporated into subsequent DE models. All code

122 and parameters used in analyses are available at,

$123 \mathrm{https}$ //github.com/awaardenberg/consensusDE_material and a comprehensive vignette

124 describing functionality: http://bioconductor.org/packages/consensusDE/ 
126 Datasets. We apply consensusDE to both real and simulated data. Real data consisted of RNA-

127 seq data comparing treatments to controls from human airway smooth muscle cells (Himes et al.

128 2014) while for simulated data, we obtain negative binomial distribution parameters from input

129 read counts of real experimental data (using the simulator described here: (Moulos \& Hatzis

130 2015)). For parameter estimation, mouse RNA-seq data comparing striatum of C57BL/6J and

131 DBA/2J strains (Bottomly et al. 2011) was obtained from recount (Frazee et al. 2011). Values

132 reported are the average of 10 simulations. We simulate DE for 10,000 genes, defining the

133 number of DE genes as 500 (5\%), with equal up/down regulation and simulate DE with 3 or 5

134 replicates.

135

136 Performance Assessment. For assessment of RNA-seq algorithm agreement or performance we 137 use a number of statistics. Agreement of $\log \mathrm{FC}$ was assessed by Bland-Altman analysis (Bland

138 \& Altman 1986) of the differences of $\log \mathrm{FC}$ versus average $\log \mathrm{FC}$ between pairs of methods, as

139 implemented in the blandr package (version 0.5.1) (Datta 2017). Bias is reported as the mean

140 shift from zero and limits of agreement defined by $95 \%$ confidence intervals. Where $R^{2}$ was

141 reported, this was the resultant goodness of fit of a linear model comparing logFC.

143 Concordance (or agreement) of significant DE genes called by RNA-seq algorithms was assessed

144 using the Jaccard Similarity coefficient (JC), (Eq. 1), representing the ratio of the size of the

145 intersecting set $A$ and $B$ to the total size or union of sets $A$ and $B$. Here, the size of set $A$ was

146 considered the intersecting set of all methods (voom, edgeR and DEseq2) and $B$ considered

147 either the set size of each method individually (where reported as either voom, edgeR or

148 DEseq2) or the union of all methods (where reported as the union).

$$
\text { Eq.1 } \quad J C=\frac{A \cap B}{A \cup B}
$$

151

152 Where truth was known (in the case of simulation), positive predictive value (PPV) or precision 153 as the proportion of true positive (TP) versus false positives (FP) (Eq. 2), sensitivity (or recall or 154 true positive rate) as the proportion of TP versus FN (Eq. 3) and False Discovery Rate (FDR) 155 (Eq. 4) were reported. 


\section{Results and Discussion}

We begin by 1) describing consensusDE functionality, followed by 2) comparison to existing software and the application to 3) real and 4) simulated data for assessing performance, with and without RUV integration. consensusDE follows two simple steps for performing DE analysis with multiple RNA-seq algorithms 1) building a summarized experiment object and 2) performing DE analysis

172 (including plotting) using the buildSummarized and multi_de_pairs functions respectively. Fig 1

173 provides an overview of a typical consensusDE workflow, and below we describe its core

174 functions.

buildSummarized. Generates a summarized experiment that contains all experimental data provided in the sample table and the read counts mapped to transcript coordinates. To build a summarized experiment object consensusDE simply requires a sample table describing the location of BAM files or pre-computed counts from the popular HTSEQ (Anders et al. 2015), sample groupings, optional pairing or technical replicate information and transcript database information (either gtf or txdb format). Where an output directory is specified, the user can save their compiled summarized experiment object for future analyses and/or reproducibility purposes. 
185 multi_de_pairs. Automatically performs DE analysis on all possible pairs of "groups" defined in 186 a provided sample table using all available DE methods (currently edgeR, voom and DESeq2)

187 and outputs a summary table (or merged table, described below) that merges the results of all

188 methods into one table. Options are provided for annotations, including an option to annotate

189 from gtf files, and users are provided the option to remove unwanted sources of variation by

190 RUVr (Risso et al. 2014). RUVr, in contrast to RUVg and RUVs (which are currently not

191 implemented in consensusDE) that rely on the assignment of negative controls genes or

192 availability of technical replicates, is readily generalisable and first fits an initial GLM fit of a

193 supervised model to estimate unknown factors of random variation, given a number of unknown

194 parameters $(\mathrm{k})$, that are subsequently incorporated into the GLM model for each algorithm for

195 estimation of transcript counts (referred to simply as RUV here after). The number of unknown

196 parameters $(\mathrm{k})$ is also fixed at 1. Options are provided for normalisation, permitting the same

197 normalisation method to be applied to RNAseq algorithm or to utilise default normalisation

198 methods for each algorithm. Full results of each method and accessibility to model details is

199 available (see vignette accompanying BioConductor package for details of how to access) and

200 where output directories are provided, results and plots are automatically written to these

201 directories, supporting batch analyses.

202

203 Merged Results. The final table of interest is described as the "merged" table (description of

204 results provided in Table 1). The merged table contains statistics including the "p_union"

205 representing the union p-value, "p_intersect" representing the intersect p-value and "rank_sum",

206 being the sum of the rankings for significance of DE reported by each method. In the case of

207 Average Expression and Log Fold Change (logFC), these represent the mean value across all

208 methods. Standard deviation of the $\log \mathrm{FC}$ is reported for assessment of variation of fold change

209 between methods. Individual p-values (corrected for multiple hypothesis testing by default), and

210 additional information including annotated gene symbol, gene name, kegg pathway and

211 chromosomal coordinates are also reported when annotation is optionally selected. Thus, the

212 merged table provides a simple summary of all methods and statistics in one location.

213

214 Plots. Ten diagnostic plots are generated and optionally saved as pdf files; 1) mapped reads for a

215 summary of transcript reads per sample, 2) Relative Log Expression (RLE) for quality control 
216 (QC) inspection, 3) Principle Component Analysis (PCA), 4) RUV residuals, 5) Hierarchical

217 clustering, 6) Density distributions, 7) Boxplot, 8) Mean/Average (MA) Plot, 9) Volcano plots

218 and 10) p-value histogram. For MA and volcano plots, the average logFC and averages of the

219 average expression by each method are used. Features are coloured by significance threshold

220 (based on the intersect p-value) and the size of the point weighted by fold change standard

221 deviation, allowing assessment of deviation of fold change by different methods. In addition to

222 providing plots before and after normalisation, if RUV is employed plots before and after RUV

223 correction are generated, thus allowing users to assess the impact of normalisation and/or RUV.

224 Each plotting function is accessible through the 'diag_plots' function in consensusDE and

225 described in a vignette that accompanies consensusDE:

226 http://bioconductor.org/packages/consensusDE/.

227

228 Software Comparison

229 For summarization of software and their features, we exclusively focus on methods that report

230 multiple RNA-seq algorithm results in S1 Table. Key criteria for software comparison were 1)

231 ease of use, 2) features, 3) correction capability and 4) integration method.

232 IDEAMEX (Jimenez-Jacinto et al. 2019) and consexpression (Costa-Silva et al. 2017)

233 implement a consensus-based voting approach, based on the number of algorithms reporting DE

234 at a pre-defined significance threshold, arguing that consensus amongst multiple methods

235 improves accuracy of DE detection. IDEAMEX, is web-based, targeted at non-bioinformaticians

236 and requires users to click through individual steps. consexpression is an instance released for

237 assessing results and is not readily generalizable. MultiRankSeq combines ranks derived from

238 edgeR, DESeq and baySeq ordered p-values as an overall rank sum for reporting of results (Guo

239 et al. 2014). Assessment of intersecting sets showed similar performance with DESeq and edgeR

240 however baySeq failed to identify a similar proportion of overlapping DE genes (Guo et al.

241 2014). All software except metaseqR were developed outside of the widely used R/Bioconductor

242 repository, requiring users to install unix-based software and in some instances install old

243 versions of software. Rather than considering intersection of common DE genes, metaseqR

244 implements several methods for combining p-values, (Simes, Union, Fisher's, and Whitlock

245 weighting) in addition to a proposed weighted PANDORA method (Moulos \& Hatzis 2015).

246 However, combining p-values using classical meta-analytical p-value methods (e.g. Fishers) 
247 performed poorly and the weighted combination of p-values (PANDORA and Whitlock) was

248 sensitive to the selection of weights, requiring proper simulation for definition of weights.

249 Although the weighted combination of $\mathrm{p}$-values improved overall performance in some cases, it

250 did not improve false discovery rate (FDR) using simulated data (which is often an important

251 goal, for example, in diagnostic settings) or Area Under Curve (AUC) of real data in comparison

252 to the intersection method (Moulos \& Hatzis 2015). MultiRankSeq and metaseqR also

253 implement DEseq, rather than DESeq2. All software requires the user to specify the contrast (or 254 comparison) and none support RUV correction.

255 In comparison, consensusDE, is available in the BioConductor repository, implements edgeR

256 (McCarthy et al. 2012; Robinson et al. 2010), DESeq2 (Love et al. 2014) and limma/voom

257 (Ritchie et al. 2015), which benchmark analyses find to be some of the best-performing

258 algorithms for DE analysis (Costa-Silva et al. 2017; Rapaport et al. 2013). A key feature of

259 consensusDE is ease of use. consensusDE does not require the user to specify a contrast of

260 interest or specify models, instead automatically performing all possible comparisons from an

261 annotation table provided. consensusDE also improves on existing methods by integrating RUV,

262 which has been reported to improve sample clustering by the removal of unwanted technical

263 variation and thereby improving biological significance (Risso et al. 2014). When selected,

264 consensusDE automatically updates the underlying model after estimating unknown sources of

265 variation from at initial GLM fit (RUVr) and including in subsequent edgeR, DESeq2 and voom

266 analyses, consistent with its goal of "ease of use". The user has the option to flag technical

267 replicates for merging of counts and paired samples (for paired analyses) in the annotation table.

268 To assess the performance of consensusDE and the utility of incorporating RUV across multiple

269 RNA-seq algorithms, we apply consensusDE with and without RUV correction to real and

270 simulated data.

271

272 Application of consensusDE to real RNA-seq data (with and without RUV)

273 RUV has been reported to stabilize fold-change and reporting of DE (Risso et al. 2014). To

274 assess if RUV improved the concordance of reported DE across different algorithms, we first use

275 the Jaccard Similarity Coefficient (JC) (Eq 1) to measure similarity of reported DE to the

276 common (intersect) set of reported DE (adjusted $\mathrm{p} \leq 0.05$ ). For assessment of RUV to stabilize

277 fold change variation between multiple algorithms, we perform Bland-Altman analysis to assess

278 bias and limits of agreement, being the mean agreement and 95\% confidence intervals of $\operatorname{logFC}$ 
279 differences between pairs of methods, respectively. Goodness of fit of log fold-change (reported 280 as $R^{2}$ ) and standard deviation (SD) of log fold-change in the intersecting and non-intersecting DE 281 results are also evaluated for each algorithm. consensusDE was ran without RUV and then with 282 RUV to incorporate the same RUV residuals into each algorithm for comparison of results. For 283 application to real RNA-seq data, we utilize data from human "airway" smooth muscle cells 284 comparing glucocorticoid treatment to untreated controls (Himes et al. 2014). This data is also 285 available in the airway R package and used as example data in the consensusDE vignette. Application of consensusDE to airway data identified 1878 DE genes for voom, 2114 for 287 edgeR and 2747 for DEseq2 (adjusted $p \leq 0.05$ ), of which 1728 were in common (Fig 2A). Voom shared the highest similarity to the intersect $(\mathrm{JC}=0.92)$, followed by edgeR $(\mathrm{JC}=0.82)$ and DEseq2 $(\mathrm{JC}=0.63)$. Application of RUV increased the overall sizes of all sets reported as DE by $18 \%$ to $31 \%$ with 2724 DE genes for voom, 2935 for EdgeR and 3341 for DEseq2 (adjusted $\mathrm{p} \leq 0.05$ ), with the common set increasing by $31 \%$ (Fig 2B). The Jaccard Similarity Coefficient similarity increased substantially for EdgeR ( 0.857 vs. 0.817$)$ and DEseq2 (0.752 vs. 0.629 ), but improved only marginally for voom ( 0.923 vs. 0.920 ) (Fig 2B-C). Therefore RUV increased the overall set size of reported DE for each algorithm (largest for voom), but also increased the intersect and JC, thus appearing to stabilize overlap. However it must be noted that an increase in JC, without known truth, does not necessarily mean improved performance. Bland-Altman analyses, linear regression and standard deviation of fold change. Bias, being the mean shift of the differences between $\operatorname{logFC}$ values for each pair of algorithms and the limit of agreement (LOA), being the range of values where $95 \%$ of data laid were compared before and after RUV correction. Mean bias, with or without RUV was small (ranging from $\sim 0.003-0.013$ ) and consistently decreased with RUV correction (S2 Table). If RUV improved agreement, we would expect the LOAs to decrease, and indeed RUV decreased the upper and lower limits of agreement between voom, DESeq2 and edgeR, but not between DESeq2 and edgeR (Figure2CD, S2 Table). DEseq2 and edgeR, however, also maintained the tightest limits of agreement irrespective of RUV . These results were consistent with correlation and standard deviation of $\operatorname{logFC}$, where $R^{2}$ improved between DESeq2 (0.9796 vs. 0.9883) and voom, and edgeR (0.9799 vs. 0.9882 ) and voom, but did not increase between DESeq2 and edgeR (0.9998 vs. 0.9999) (S2 
310 change SD improved for each method, 0.013 (reduction of 0.004 ) for voom, 0.016 (reduction of

3110.005 ) for EdgeR, and 0.013 (reduction of 0.003) for DEseq2 (adjusted $p \leq 0.05$ ). The non-

312 intersecting set for each algorithm also improved, 0.008 (reduction of 0.004) for voom, 0.027

313 (reduction of 0.012) for EdgeR and 0.012 (reduction of 0.007) for DEseq2 and intersection 0.014

314 (reduction of 0.003). Only voom demonstrated lower fold change SD than the intersecting set

315 compared to DEseq2 and EdgeR which exhibited higher fold change SD.

316 Overall, these results found that RUV 1) increased the number of reported DE genes,

317 whilst 2) increasing the overlap of intersecting sets and 3) improving fold change agreement

318 between methods. Thus, RUV appeared to improve the overall concordance of different

319 methods, bringing their intersecting values closer to the union of all sets. This was consistent

320 with a reduction of variability of fold change between algorithms, especially between voom and

321 DEseq2 as well as edgeR. However, the smallest set of DE genes was also derived from voom

322 and voom had the highest level of agreement to the overall intersect. Thus, it is important to note

323 that consensus and improvement of consensus with RUV, was largely driven by voom and the

324 additional agreement of DEseq2 and edgeR with voom after RUV application. As it is difficult to

325 address what this means, especially JC, in the context false discovery rate or overall

326 performance, we next simulate data with known numbers of DE genes and assess the utility of

327 applying RUV in a consensus-based manner across multiple RNA-seq algorithms for improving

328 stability of the intersecting set.

329

330 Simulation Results.

331 For simulated data (described in Methods) we set an expected number of DE genes to 500 (or

$3325 \%$ ), from a total of 10,000 genes with equal up/down regulation between two groups. We

333 simulate DE with 3 and 5 replicates (in equal groups), representing commonly performed

334 experimental designs, and report the average of 10 simulations. As per the real "airway" dataset,

335 we begin by assessing the JC of each method, with and without RUV correction, followed by

336 Bland-Altman analysis of bias, LOA, $R^{2}$ and fold change SD. Simulated data with 3 replicates

337 (without RUV and adjusted $\mathrm{p} \leq 0.05$ ) identified 304.0 DE genes for voom (JC = 0.999), 449.6

338 for edgeR $(\mathrm{JC}=0.675), 438.1$ for DEseq2 $(\mathrm{JC}=0.693)$, of which 303.6 were in common. In

339 contrast to real data RUV did not improve JC values, identifying 281.9 DE genes for voom (JC =

$3400.996), 462.2$ for edge $(\mathrm{JC}=0.608)$ and 415.5 for DEseq2 $(\mathrm{JC}=0.676)$, of which 280.8 were in 
341 common (Fig 3A and S2 Table). Although JC improved overall for all methods when increasing

342 to 5 replicates, JC was once again not improved with the application of RUV (Fig 3A). For 5

343 replicates, comparing RUV vs non-RUV corrected, this identified 413.1 vs. 415.5 (JC $=0.997$

344 vs. 0.996) DE genes for voom, 487.6 vs. 481.8 (JC=0.845 vs. 0.859) for edgeR and 492.8 vs.

$345492.0(\mathrm{JC}=0.837$ vs. 0.841$)$ for DEseq2 (adjusted $\mathrm{p} \leq 0.05)$, of which 413.8 vs. 411.8 were in

346 common. Thus, replication rather than application of RUV had the greatest impact on JC in

347 simulated data.

348 In contrast to the real data and albeit that the differences were negligible (a maximum

349 mean difference of 0.008), RUV increased the mean absolute fold-change bias in all comparisons

350 (S2 Table). However, consistent with the real airway data, upper and lower limits of agreement

351 for simulated data decreased with RUV correction for voom comparisons with DESeq2 and

352 edgeR but not between DESeq2 and edgeR (Fig 3B, S2 Table). Notably, limits of agreement

353 were consistently lower for simulations with 5 replicates (Fig 3B, S2 Table). Overall fold change

$354 R^{2}$ for 3 and 5 replicates also improved between DESeq2 and voom, as well as edgeR and voom,

355 but did not increase between DESeq2 and edgeR (S2 Table).

356 The greatest gains in fold change stability for voom were found in the 3-replicate setting,

357 doubling the improvement observed with RUV applied in a 5-replicate setting. Absolute mean

358 fold change SD of simulated results also improved with RUV correction, 0.0086 (vs 0.011 or a

359 reduction of 0.024 ) for voom, 0.0147 (vs 0.0154 or a reduction of 0.007 ) for edgeR, and 0.0133

360 (vs 0.0141 or a reduction of 0.008 ) for DEseq2 (adjusted $\mathrm{p} \leq 0.05$ ). With the exception of

361 edgeR, the non-intersecting set of each method and the overall intersect also improved with 5

362 replicates, which was 0.0534 (vs. 0.0315, an increase of 0.029) for voom, 0.0242 (vs 0.0252 , a

363 decrease of 0.01) for EdgeR and 0.0232 (vs 0.0217, an increase of 0.015) for DEseq2 and

364 intersection ( 0.0085 vs 0.0107 , a decrease of 0.022) (S2 Table). RUV also reduced mean

365 absolute SD with 5 replicates and the intersect had the lowest value (0.0085), but increasing to 5

366 replicates did not improve overall deviation of fold change, compared to 3 replicates. Thus, the

367 greatest gains of RUV were made in a setting of 3-replicates, with diminished improvement for

368 voom with additional replication.

369 As we defined a truth set (known DE genes) we assess the performance of consensusDE

370 with and without RUV correction for each method, as well as the union and intersect. We utilise

371 1) False Discovery Rate (FDR) (Fig 3C, Eq 4) and 2) sensitivity (or recall) (Fig 3D, Eq 3) for 
372 assessment of false positive and false negative rates. Mean FDR (of 10 simulations) was lowest

373 for the intersect and improved with correction of RUV (0.0491 vs. 0.0384 , for 3 replicates). FDR

374 improved with the application of RUV for voom (0.0494 vs 0.0390$)$ and DEseq2 (0.1654 vs

375 0.1565), but it did not improve for the union ( 0.1811 vs 0.1918$)$ or edgeR ( 0.1610 vs 0.1847$)$

376 (Fig 3C). Although the same pattern emerged for the FDR of 5 replicates, whereby the intersect

377 had the lowest FDR (0.0405), application of RUV did not improve the FDR for any individual

378 method (S2 Table). Although a low FDR is desirable in some instances, ensuring the number of

379 false positives are minimised, controlling for false negatives whereby true results are incorrectly

380 missed (or recall/sensitivity) can also be of importance. In contrast to FDR, sensitivity did not

381 increase with the application of RUV for any algorithm tested, indeed decreasing with RUV

382 application and was highest in a setting of 5 replicates for the union of all methods (0.872).

383 Here, we establish that RUV improved FDR for the intersect for 3 replicates, approaching

384 the same FDR as 5 replicates without application of RUV, but did not further improve FDR with

3855 replicates. The improvement of FDR related to a reduction of fold-change variation and limit

386 of agreement of different algorithms, which was greater in a setting of 3 replicates versus 5

387 replicates. However, the same was not true for all algorithms individually, suggesting that the

388 greatest gain in FDR results from the combination of different algorithms. Observing the lowest

389 FDR for the intersect amongst different algorithms and the highest for the union could be

390 considered consistent with a model where increased stringency through inclusion of multiple

391 forms of evidence improves FDR. Indeed, this would be consistent with previous benchmarking

392 studies (Costa-Silva et al. 2017; Guo et al. 2014; Moulos \& Hatzis 2015). However, we found

393 that voom was also the most conservative method, based on set size of DE, in both the 3 and 5

394 replicate experiments and that voom FDR was also the lowest. Hence voom was the main driver

395 of the low FDR observed in the intersect. This is consistent with previous results demonstrating a

396 lower proportion of false positive results by voom (Ritchie et al. 2015). Overall, RUV improved

397 the confidence of identifying true positives, or minimizing false positives, when combined with

398 the intersect of DE reported by multiple algorithms and experiments performed with a lower

399 number of replicates, but with minimal gains over using voom alone. Importantly, the difference

400 between FDR performance with the application of RUV to lower numbers of replicates (3 versus

401 5) diminished with increased replication. This was likely due to the improved performance of the

402 linear model based voom without RUV correction. Although a trade-off between recall and 
403 precision was observed for voom, with voom also being the least sensitive, RUV did not improve 404 sensitivity for any method, in 3 or 5 replicate setting. Indeed the union, being the combination 405 (rather than intersection) of all evidence, performed better than any other individual method.

406 This suggests that RUV is favouring improvement of FDR (or precision) through minimising 407 false positives, but potentially at the cost of false negatives. In this context, we also note that 408 increased JC observed is a poor metric for assessment of FDR. Real data was not replicated with 409 our simulated data, where instead a decreased JC coincided with improved FDR. However, RUV 410 certainly improved $\log \mathrm{FC}$ agreement between methods, especially between voom and edgeR as 411 well as DEseq2. Overall, these results support the application of RUV in a low-replicate setting 412 for stabilisation of fold-change, in particular for when voom and DEseq2, however RUV did not 413 improve FDR with increased replication or sensitivity in any setting.

\section{Conclusions}

416 We present consensusDE, a freely available R/Bioconductor package, that allows for simple and 417 automated DE analysis to be performed using multiple methods, readily allowing the user to 418 observe variability of DE due to method selection. Application of consensusDE to real and 419 simulated data highlights the following rules, some of which are already established in the RNA420 seq community and others that require further consideration when analysing RNA-seq data. 1) 421 the intersect of multiple methods has lowest FDR (but this can be driven by a single high 422 performing algorithm, which we find to be voom), 2) RUV improves the intersect FDR (as well 423 as voom and DESeq2 individually) with smaller number of replicates but this effect diminishes 424 with increased replication, 3) RUV does not improve FDR for all individual RNA-seq 425 algorithms, 4) RUV does not improve sensitivity, 5) the union appears to strike a balance for 426 recovery of true positives, through minimising loss through false negatives, when using multiple 427 methods, and finally 6) increased replicate numbers, without RUV, has the best recovery of DE 428 genes when considering FDR and sensitivity together - reinforcing increased replication for 429 recovery of true DE genes. We do note however that this is not an exhaustive testing of all 430 possible scenarios. For instance, it remains to be explored if these rules apply with increased 431 modelling of noise, RUV correction methodology (here we apply RUVr), number of hidden 432 variables or if these rules generalize to other methods for combining RNA-seq algorithms, such 433 as weighting of p-values. However, our results do indicate the utility and ease of consensusDE 
434 for performing analysis with multiple RNA-seq algorithms and integration with RUV, whilst

435 offering some rules for considering results. Future work will aim to incorporate additional

436 algorithms and combination methods.

437

438

439

440

441

442

443

444

445

446

447

448

449

450

451

452

453

454

455

456

457

458

459

460

461

462

463

464

465

466

467

468

469

470

471

472

473

474

475

476

477

478

479

\section{References}

Anders S, Pyl PT, and Huber W. 2015. HTSeq--a Python framework to work with highthroughput sequencing data. Bioinformatics 31:166-169. 10.1093/bioinformatics/btu638

Bland JM, and Altman DG. 1986. Statistical methods for assessing agreement between two methods of clinical measurement. Lancet 1:307-310.

Bottomly D, Walter NA, Hunter JE, Darakjian P, Kawane S, Buck KJ, Searles RP, Mooney M, McWeeney SK, and Hitzemann R. 2011. Evaluating gene expression in C57BL/6J and DBA/2J mouse striatum using RNA-Seq and microarrays. PLoS One 6:e17820. 10.1371/journal.pone.0017820

Costa-Silva J, Domingues D, and Lopes FM. 2017. RNA-Seq differential expression analysis: An extended review and a software tool. PLoS One 12:e0190152. 10.1371/journal.pone.0190152

Datta D. 2017. blandr: a Bland-Altman Method Comparison package for R. Available at https://github.com/deepankardatta/blandr.

Delhomme N, Padioleau I, Furlong EE, and Steinmetz LM. 2012. easyRNASeq: a bioconductor package for processing RNA-Seq data. Bioinformatics 28:2532-2533. 10.1093/bioinformatics/bts477

Frazee AC, Langmead B, and Leek JT. 2011. ReCount: a multi-experiment resource of analysis-ready RNA-seq gene count datasets. BMC Bioinformatics 12:449. 10.1186/1471-2105-12-449

Gentleman RC, Carey VJ, Bates DM, Bolstad B, Dettling M, Dudoit S, Ellis B, Gautier L, Ge Y, Gentry J, Hornik K, Hothorn T, Huber W, lacus S, Irizarry R, Leisch F, Li C, Maechler M, Rossini AJ, Sawitzki G, Smith C, Smyth G, Tierney L, Yang JY, and Zhang J. 2004.

Bioconductor: open software development for computational biology and bioinformatics. Genome Biol 5:R80. 10.1186/gb-2004-5-10-r80

Guo Y, Zhao S, Ye F, Sheng Q, and Shyr Y. 2014. MultiRankSeq: multiperspective approach for RNAseq differential expression analysis and quality control. Biomed Res Int 2014:248090. 10.1155/2014/248090

Himes BE, Jiang X, Wagner P, Hu R, Wang Q, Klanderman B, Whitaker RM, Duan Q, Lasky-Su J, Nikolos C, Jester W, Johnson M, Panettieri RA, Jr., Tantisira KG, Weiss ST, and Lu Q. 2014. RNA-Seq transcriptome profiling identifies CRISPLD2 as a glucocorticoid responsive gene that modulates cytokine function in airway smooth muscle cells. PLoS One 9:e99625. 10.1371/journal.pone.0099625

Jimenez-Jacinto V, Sanchez-Flores A, and Vega-Alvarado L. 2019. Integrative Differential Expression Analysis for Multiple EXperiments (IDEAMEX): A Web Server Tool for Integrated RNA-Seq Data Analysis. Front Genet 10:279. 10.3389/fgene.2019.00279

Lin B, and Pang Z. 2019. Stability of methods for differential expression analysis of RNA-seq data. BMC Genomics 20:35. 10.1186/s12864-018-5390-6

Love MI, Huber W, and Anders S. 2014. Moderated estimation of fold change and dispersion for RNA-seq data with DESeq2. Genome Biol 15:550. 10.1186/s13059-014-0550-8

Peer) reviewing PDF | (2019:08:40512:1:0:NEW 30 Oct 2019) 
480

481

482

483

484

485

486

487

488

489

490

491

492

493

494

495

496

497

498

499

500

501

502

503

504
McCarthy DJ, Chen Y, and Smyth GK. 2012. Differential expression analysis of multifactor RNA-Seq experiments with respect to biological variation. Nucleic Acids Res 40:42884297. 10.1093/nar/gks042

Moulos P, and Hatzis P. 2015. Systematic integration of RNA-Seq statistical algorithms for accurate detection of differential gene expression patterns. Nucleic Acids Res 43:e25. 10.1093/nar/gku1273

Rapaport F, Khanin R, Liang Y, Pirun M, Krek A, Zumbo P, Mason CE, Socci ND, and Betel D. 2013. Comprehensive evaluation of differential gene expression analysis methods for RNA-seq data. Genome Biol 14:R95. 10.1186/gb-2013-14-9-r95

Risso D, Ngai J, Speed TP, and Dudoit S. 2014. Normalization of RNA-seq data using factor analysis of control genes or samples. Nat Biotechnol 32:896-902. 10.1038/nbt.2931

Ritchie ME, Phipson B, Wu D, Hu Y, Law CW, Shi W, and Smyth GK. 2015. limma powers differential expression analyses for RNA-sequencing and microarray studies. Nucleic Acids Res 43:e47. 10.1093/nar/gkv007

Robinson MD, McCarthy DJ, and Smyth GK. 2010. edgeR: a Bioconductor package for differential expression analysis of digital gene expression data. Bioinformatics 26:139140. 10.1093/bioinformatics/btp616

Seyednasrollah F, Laiho A, and Elo LL. 2015. Comparison of software packages for detecting differential expression in RNA-seq studies. Brief Bioinform 16:59-70. 10.1093/bib/bbt086

Soneson C, and Delorenzi M. 2013. A comparison of methods for differential expression analysis of RNA-seq data. BMC Bioinformatics 14:91. 10.1186/1471-2105-14-91

Varet H, Brillet-Gueguen L, Coppee JY, and Dillies MA. 2016. SARTools: A DESeq2- and EdgeR-Based R Pipeline for Comprehensive Differential Analysis of RNA-Seq Data. PLoS One 11:e0157022. 10.1371/journal.pone.0157022 


\section{Figure 1}

consensusDE typical workflow

(A) consensusDE requires a table, here defined as "my_data", for example purposes, that describes the experimental design and location of files. (B) running consensusDE requires two steps, first to build a summarized object, using the buildSummarized function, to store all information and second to run analyses with all algorithms using the multi_de_pairs function. Example code for a typical analysis with consensusDE is provided for illustration. 


\section{A. Description of data}

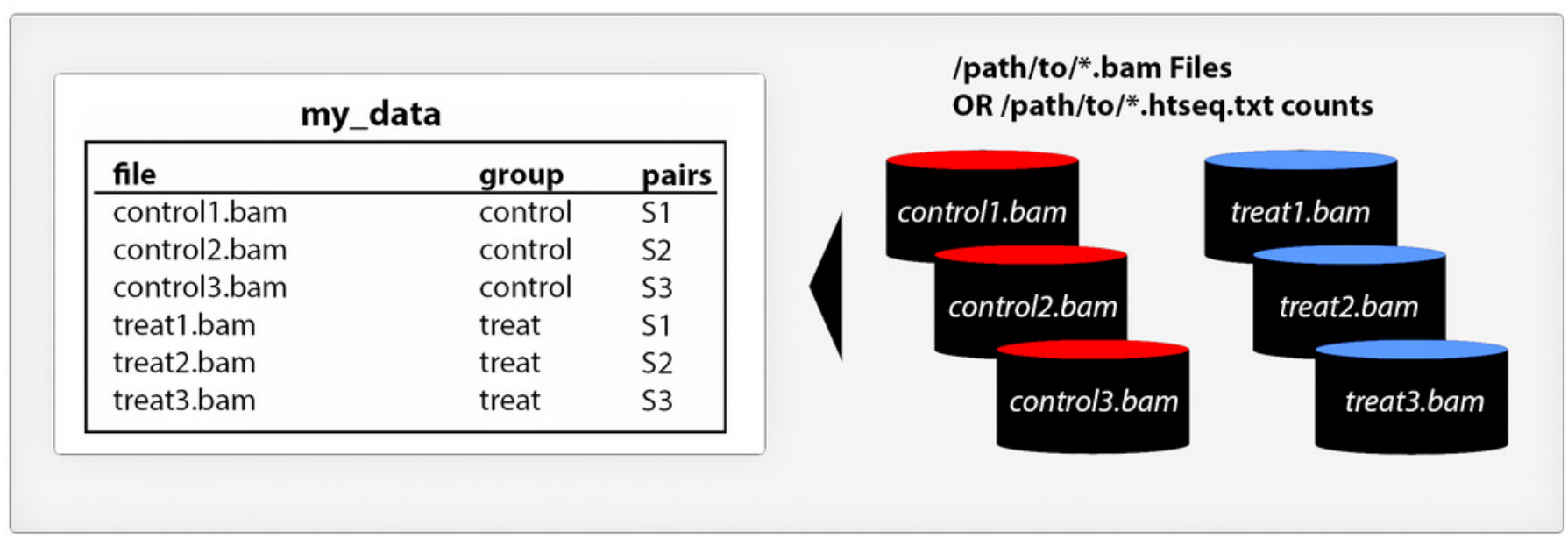

\section{B. consensusDE workflow}

\section{STEP 1 - buildSummarized}

generate summarized experiment object

1) describes experiment

2) stores reads mapped to features

\section{STEP 2 - multi_de_pairs}

perform $D E$ on all pairs of samples in table provided

1) edge $R+$ voom + DEseq 2

2) produce $10 x$ plots

3) optional RUV correction

4) annotate results

\section{\# EXAMPLE CODE}

\# Step 1

my_summarized <- buildSummarized(

sample_table $=$ my_data, bam_dir="/path/to/my_data/", tx_db $=$ tx_db_object)

\# Step 2

de_results <- multi_de_pairs(

summarized = my_summarized)

\# access merged results for all algorithms

de_results\$merged 
Figure 2

Application to airway data

(A) Jaccard Coefficient (JC) of each set to the intersect (common) without RUV correction and

(B) with RUV correction. Absolute upper (C) and lower (D) limits of log fold change differences between pairs of algorithms (95\% confidence intervals), with and without RUV correction.

\section{A. Overlap (w/out RUV)}

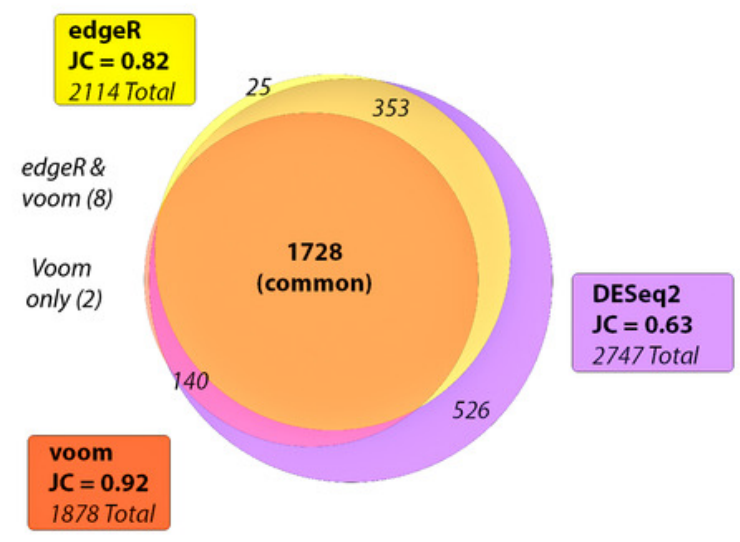

\section{Upper limit of logFC agreement}

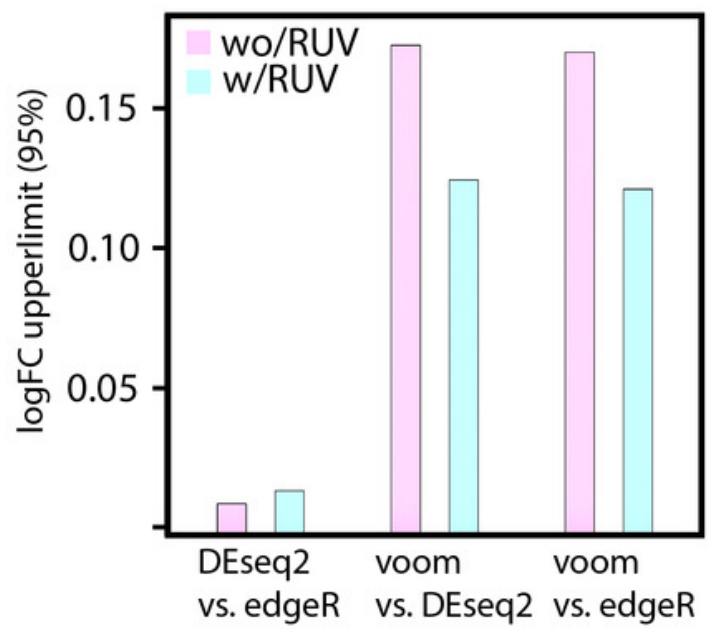

\section{B. Overlap (w/RUV)}

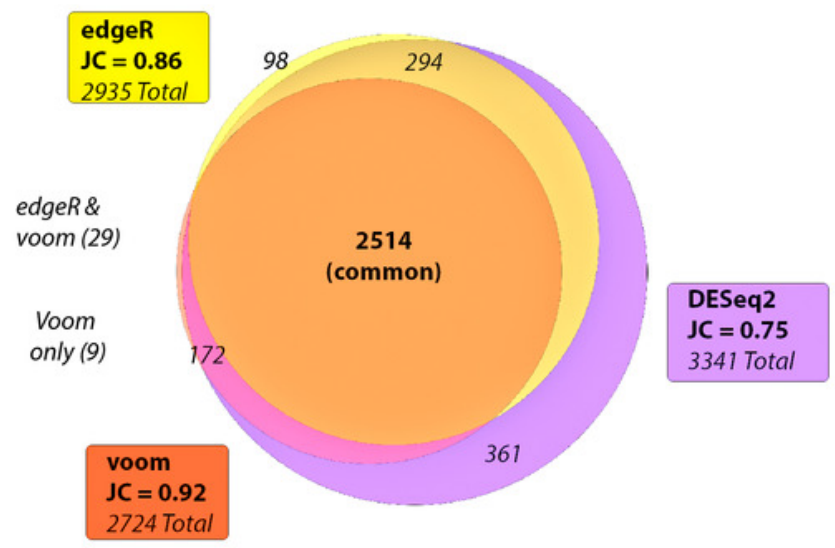

\section{Lower limit of logFC agreement}

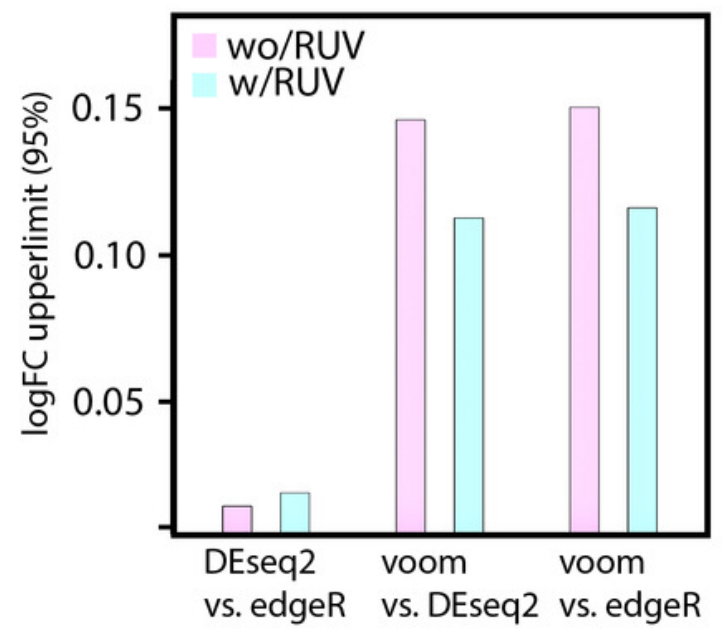




\section{Figure 3}

Simulated data for 3 and 5 replications with and without RUV

(A) Jaccard Coefficient (JC) of each method (B) limits of log fold change differences between pairs of algorithms (95\% confidence intervals), with and without RUV correction (C) False Discovery Rates (FDR), lower number is better and (D) Sensitivity (or recall), higher number is better. Each panel contains 3 replicates, 5 replicates and without and without RUV correction - see central legend for colour coding scheme. All values represent the absolute average of 10 simulations. 


\section{A. Jaccard Coefficient}
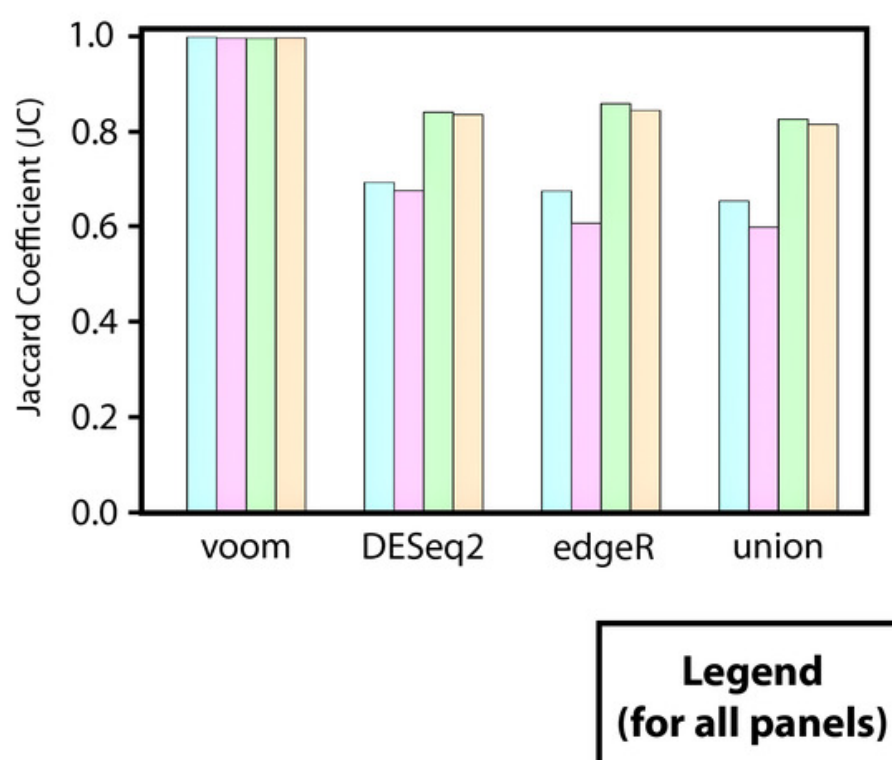

C. False discovery rate (FDR)

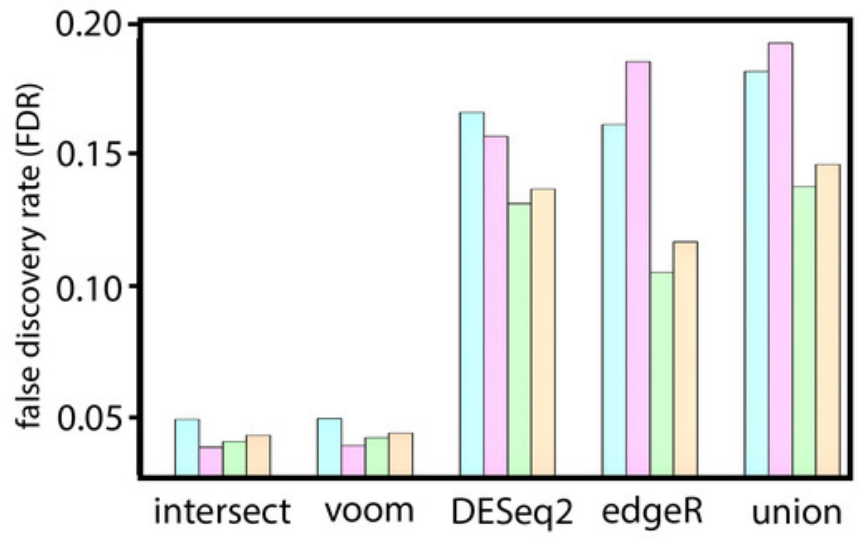

B. Limits of agreement (95\% LOA)

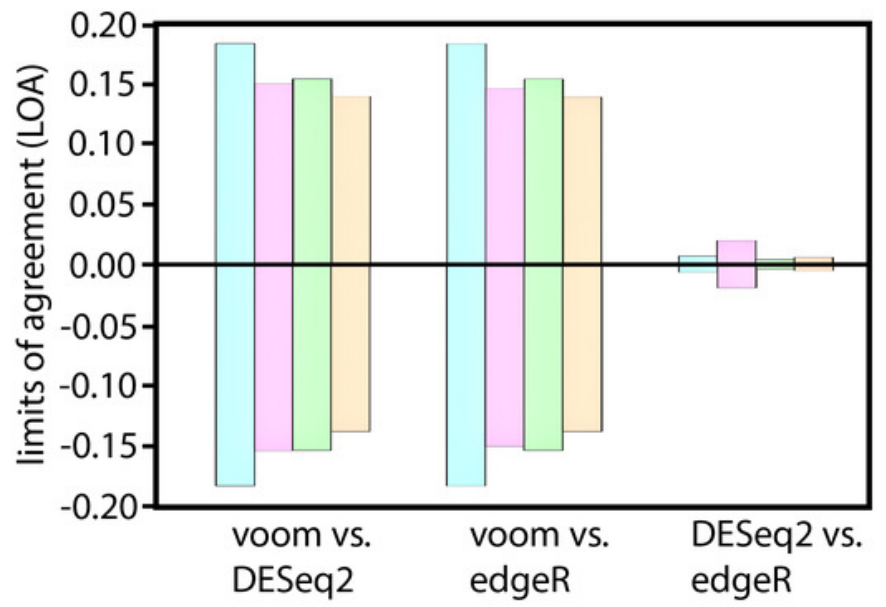

3 rep wo/RUV 3 rep w/RUV 5 rep wo/RUV $5 \mathrm{rep}$ w/RUV

D. Sensitivity

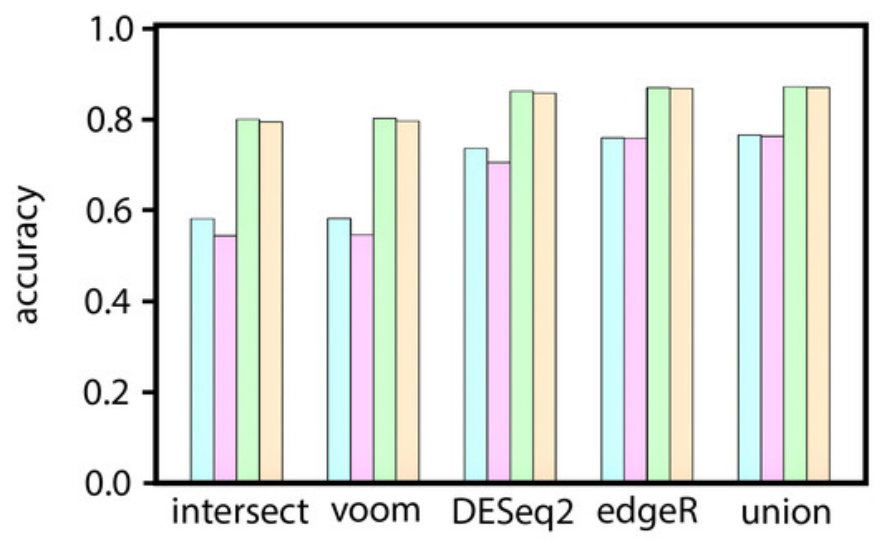




\section{Table $\mathbf{1}$ (on next page)}

consensusDE merged table features and description 


\begin{tabular}{|c|c|c|}
\hline Reported Feature & Meaning & Description \\
\hline ID & Identifier & $\begin{array}{l}\text { Indentified of feature used for } \\
\text { mapping read counts against }\end{array}$ \\
\hline AveExpr & Average Expression & $\begin{array}{l}\text { Average of edgeR, DESeq } 2 \text { and } \\
\text { voom reported Average Expression }\end{array}$ \\
\hline LogFC & $\begin{array}{l}\text { Log Fold-Change, also known as a log- } \\
\text { ratio }\end{array}$ & $\begin{array}{l}\text { Average of edgeR, DESeq } 2 \text { and } \\
\text { voom logFC }\end{array}$ \\
\hline LogFC_sd & Log Fold-Change standard deviation & $\begin{array}{l}\text { Standard Deviation of LogFC } \\
\text { reported by edgeR, DESeq } 2 \text { and } \\
\text { voom }\end{array}$ \\
\hline edger_adj_p & edgeR $p$-value & $\begin{array}{l}\text { Adjusted for multiple hypotheses } \\
\text { using benjamini and hochberg } \\
\text { (default) }\end{array}$ \\
\hline deseq_adj_p & DESeq2 $p$-value & $\begin{array}{l}\text { Adjusted for multiple hypotheses } \\
\text { using benjamini and hochberg } \\
\text { (default) }\end{array}$ \\
\hline voom_adj_p & voom p-value & $\begin{array}{l}\text { Adjusted for multiple hypotheses } \\
\text { using benjamini and hochberg } \\
\text { (default) }\end{array}$ \\
\hline edger_rank & rank of the $p$-value reported by edgeR & $\begin{array}{l}\text { smallest rank is most significant (or } \\
\text { smallest } p \text {-value) reported }\end{array}$ \\
\hline deseq_rank & rank of the $p$-value reported by DESeq 2 & $\begin{array}{l}\text { smallest rank is most significant (or } \\
\text { smallest } p \text {-value) reported }\end{array}$ \\
\hline voom_rank & rank of the $p$-value obtained by voom & $\begin{array}{l}\text { smallest rank is most significant (or } \\
\text { smallest } p \text {-value) reported }\end{array}$ \\
\hline rank_sum & Sum of ranks & $\begin{array}{l}\text { Combination of ranks from } \\
\text { edger_rank, voom_rank, rank_sum } \\
\text { Results are orderd by this sum, } \\
\text { which represents the order of the } \\
\text { most stable reported p-values }\end{array}$ \\
\hline p_intersect & Largest $p$-value observed & $\begin{array}{l}\text { This represents the intersect when } \\
\text { a threshold is set on the } \\
\text { p_intersect column }\end{array}$ \\
\hline p_union & Smallest p-value observed & $\begin{array}{l}\text { This represents the union when a } \\
\text { threshold is set on the p_union } \\
\text { column }\end{array}$ \\
\hline
\end{tabular}




\begin{tabular}{|l|l|l|} 
& & $\begin{array}{l}\text { e.g. alpha-L-fucosidase 2 } \\
\text { corresponds to FUCA2 }\end{array}$ \\
\hline symbol & Extended gene name & e.g. FUCA2 \\
\hline kegg & Gene symbol & $\begin{array}{l}\text { For further analyses of pathways } \\
\text { where annotated }\end{array}$ \\
\hline coords & kegg pathway identifier & e.g. chr6:143494811-143511690 \\
\hline strand & chromosomal coordinates & $\begin{array}{l}\text { forward strand is +, reverse strand } \\
\text { is - }\end{array}$ \\
\hline & transcript strand & $\begin{array}{l}\text { Reported in base pairs (bp) } \\
\text { (transcript start to end) (e.g. } 16880 \\
\text { bp) }\end{array}$ \\
\hline width & &
\end{tabular}

1 